\title{
2D vs. 3D Pain Visualization: User Preferences in a Spinal Cord Injury Cohort
}

\author{
Fotios Spyridonis $^{1}$ and Gheorghita Ghinea ${ }^{1}$ \\ ${ }^{1}$ Department of Information Systems and Computing, Brunel University, Uxbridge, UK \\ \{Fotios.Spyridonis, George.Ghinea\}@brunel.ac.uk
}

\begin{abstract}
Research on pain experienced after Spinal Cord Injury (SCI) has revealed that not only are there several types of pain present in the same individual with this kind of trauma, but also that people who suffer such an injury can describe the characteristics of the same type of pain in different ways. Making it possible, therefore, to more precisely describe pain experience could prove to be vital for an increased quality of life. Accordingly, fifteen individuals with pain after SCI were asked to describe their pain experience using a 3 Dimensional (3D) model of the human body that could be used as an aid in communicating their pain. The results of this study suggest that the consensus of the participants approved the ability of the 3D model to more accurately describe their pain, an encouraging outcome towards the use of 3D technology in support of post SCI pain rehabilitation.
\end{abstract}

Keywords: Rehabilitation, Chronic Pain, Spinal Cord Injury, 3D Visualization, Pain Drawing

\section{$1 \quad$ Introduction}

Studies on the implications of Spinal Cord Injury on patients have exposed that post traumatic pain is amongst its most prevalent consequences, coming only second after loss of function. Reports from individuals who have suffered this kind of trauma reveal that the severity of such pain experience could be so immense that it often interferes with sleep and everyday activities [4]. In fact, a summary of results from several studies [see 16,19] indicates that the average reported estimate of the prevalence of chronic SCI pain is approximately $65 \%$, with roughly one-third of those affected reporting the severity as greater than 7 in a scale of 10 on a Visual Analogue Scale (VAS). This revelation could justify the need of the nearly $40 \%$ of SCI patients to trade their pain with additional loss of mobility, bladder or bowel control, and sexual function [16].

As a result of its incidence, considerable research efforts towards pain relief have been reported so far [7, 9]. Nevertheless, the majority of them have been criticized in the clinical literature regarding their applicability in assessing persons with SCI. The reason behind this controversy lays in the multidimensional nature of pain, which is characterized by physical discomfort, and is often influenced by complex qualities 
associated with psychological and cultural factors [3, 10, 13]. Owing to its subjectivity, it is therefore argued, that individuals who have to deal with pain after SCI may frequently experience substantial difficulties when it comes to precisely describe their pain characteristics, as they may have been influenced by the above factors, resulting in different interpretations of the same pain experience.

To this end, considering the heterogeneity of the pain experience in this patient population, enabling the individual to also visually communicate his/her pain was further introduced as a supplementary approach for the intended purpose. Accordingly, the focus of the work described in this paper has been to examine from the user perspective the applicability of a $3 \mathrm{D}$ visualization approach in the communication of pain, in the anticipation that it will constitute a significant improvement over the current approaches and make an important contribution towards the effective assessment of pain for persons with SCI. Subsequently, the structure of this paper is as follows: Section 2 presents an overview of the current methods used for pain visualization, while section 3 discusses the methodology used to evaluate our 3D model as compared to the current approaches. Finally, section 4 presents the results of our study, and section 5 concludes with our study's discussion.

\section{Current Approaches to Pain Visualization}

The application of 2-Dimensional (2D) visualization techniques in the assessment of pain is not a recent trend. Over the last decades, a simple self-assessment method the 'pain drawing' (Fig. 1) - has been exponentially applied across several medical conditions in the attempt to assist patients to provide their pain information. Originally proposed in the 1940 s by $[12,14]$, this technique is considered to be a popular visual aid tool among the clinical field, as it enables patients to describe, using a monochrome symbol, the spatial location, as well as the type (usually ache, pain, pins and needles, and numbness) of their pain on a $2 \mathrm{D}$ human diagram. Considering its wide applicability, a number of research studies have been conducted over the years to examine its validity for the intended purpose. Their results indicate that the pain drawing is considered to be a valuable and useful tool in identifying pain location and sensation type, with most of patients demonstrating consistency in completing it $[12,18]$.

Due to their established usefulness and acceptance in assessing pain across various medical conditions $[8,11,12]$, there have also been efforts to utilise the benefits of pain drawings to the SCI population that is characterised by the severity of this injury, which appears, for instance, in approximately more than 1,000 people per year in Britain alone[17]. 


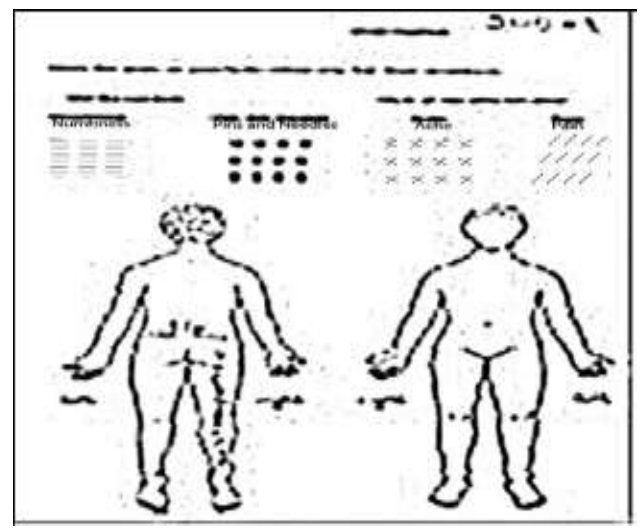

Fig. 1. 2D Pain Drawing.

To this end, [15] have used pain drawings to assess back pain and spinal deformity, whereas [4] attempted to identify and relieve the most disturbing pains in patients with a SCI that affect the quality of their life. Similarly, [5] utilised pain drawings before and after surgery to evaluate treatment interventions in SCIs.

\subsection{Exploring the Need for 3D in the Assessment of SCI Pain}

This situation, however, is changing rapidly. Advances in computer graphics technology towards a 3-Dimensional perception of the environment offer the ability for a more accurate and interactive experience. As a result, recent technological advancements have enabled the use of such 3D visualization expertise in offering promising opportunities for clinical rehabilitation. For instance, the use of 3D interactive representations of the head and arm has shown to be beneficial for patients in terms of enhancing their motor performance after a stroke [1], while innovations in $3 \mathrm{D}$ techniques have enabled the reconstruction of facial prosthesis for cancer patients [2]. The visualization capabilities that $3 \mathrm{D}$ technology provides could be similarly exploited towards the design of novel assistive rehabilitation solutions for persons with pain after Spinal Cord Injury.

To this end, after consultations with medical staff in the Royal National Orthopaedic Hospital in London, UK, it was considered more beneficial that patients would be offered the ability to describe the pain that they were experiencing onto a $3 \mathrm{D}$ model of themselves rather than a $2 \mathrm{D}$ one, as the former provides a continuous body surface, which allows patients to better map their pain. As a result, by expanding on past research, an adaptation of the 3D pain drawing (Fig. 2b) that was initially devised by [6] (Fig. 2a) was developed. This revised 3D model was considered to be more suitable for the purpose intended, in the anticipation that it would remove any anomalies caused by the limited depth perception of the 2D pain drawing currently used in SCI pain assessment, while addressing statements of the form "I have a pain on the inside of my thigh", which are not easily captured in a 2D visualization of pain. 
Accordingly, while the concept of 2D drawings is well-established amongst the pain community, the usefulness of their $3 \mathrm{D}$ equivalent is yet to be examined. In this paper, therefore, we report the results of an empirical study, which aimed to identify the user preferences regarding the most well perceived way of visualizing their pain, as derived by comparing both the $2 D$ and the $3 D$ methods while used in a natural clinical setting.

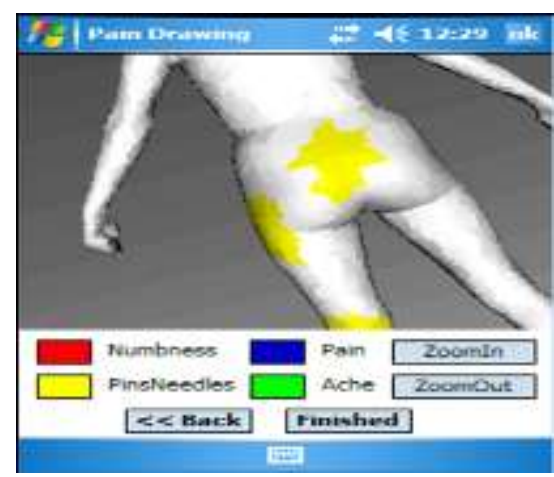

Fig. 2a. Initial 3D Pain Drawing.

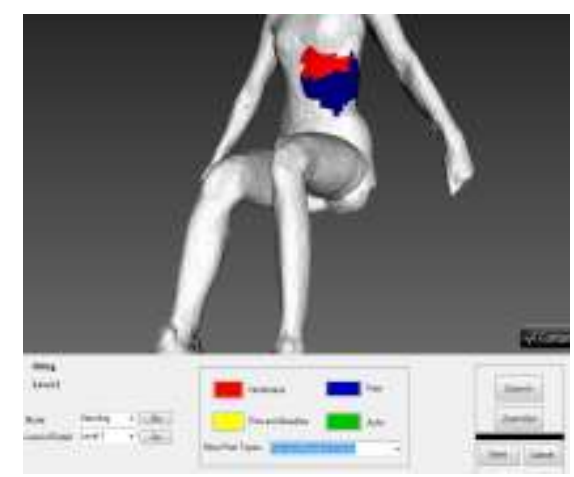

Fig. 2b. 3D Pain Drawing for SCIs.

\section{Methodology}

Consequently, the objectives of this study are: $a$. to investigate the feasibility of the $3 D$ model in visualizing patient SCI pain characteristics, as well as $b$. to examine its usability in achieving the above. To keep with best practice, our developed 3D model will be evaluated against the well-established 2D pain drawing of Fig. 1. Therefore, to address our research objectives, each participant will be given the chance to use both methods and at the end fill an evaluation questionnaire about the feasibility and usability of using these two methods for the purpose of recording and visualizing their pain experience.

\subsection{Instrumentation and Materials}

The instrumentation used for this study consists of a laptop that runs the 3D pain drawing, the paper-based 2D pain drawing, and an evaluation questionnaire. The aforementioned questionnaire is an evaluation survey for both the 2D and 3D pain drawings, in which patients are asked to record their opinions about both methods on a Likert scale of 1 (Disagree) to 5 (Agree) (Table 1 below). 
Table 1. Participant Evaluation Questionnaire.

\begin{tabular}{|l|}
\hline \multicolumn{1}{|c|}{ Questions } \\
\hline Q1. It was easy to log pain information on the pain diagram \\
\hline Q2. Showing the exact type and location of my pain on the pain drawing was easy \\
\hline Q3. I believe the pain drawing was insufficient to express my pain \\
\hline Q4. How would you describe the overall layout of the interface? \\
\hline
\end{tabular}

\subsection{Description of Subject Group}

The research sample consisted of 15 persons with SCI ( 7 female; 8 male, mean age 52.3 years, range 28-75) who volunteered to participate in the research study between July 2010 and October 2010. All were recruited from the Spinal Cord Injury Unit in the Royal National Orthopedic Hospital, London, UK. The diagnosis varied, but the majority was identified with traumatic SCI. The criteria for selection was that the participant has spinal cord trauma, has an age of 20 years or greater and experiences some pain during the period of study. Finally, the range of pain intensity varied from $0-9$, with the mean maximum pain intensity being 8.375 on a Visual Analogue Scale (VAS)

\subsection{Evaluation Protocol}

After consultations with the clinicians, it was decided that the data collection would take place in four points in time over a period of one day for each participant, with an approximately 2-3 hour time difference between these four recordings (between $8.30 \mathrm{am}$ and $5 \mathrm{pm}$ ) in order to address the change of pain over time. For the first two recordings, the participant was asked to report any pain experience on the paperbased, 2D pain drawing, whereas for the remaining 2 recordings s/he was similarly asked to report any pain on the laptop-based, 3D pain drawing provided. Each session lasted approximately 20 minutes. At the end of the first two sessions, the participant filled in the evaluation questionnaire for the 2D pain drawing. Accordingly, the same questionnaire evaluating the 3D pain drawing was also provided after the end of the last, fourth session. Finally, another participant would be approached on the next scheduled day, and the protocol was repeated.

\section{$4 \quad$ Evaluation Results and Discussion}

The analysis of the results consisted of a graphical investigation performed by using statistical software such as Microsoft Excel, and highlighted a general consensus (Fig. 3) that the participants had in respect to the ability of the 3D pain drawing to better visualize and record their pain, as compared to the $2 \mathrm{D}$ pain drawing. 


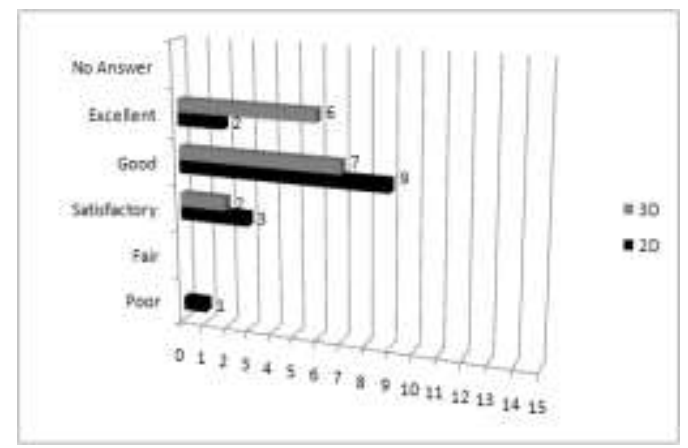

Fig. 3. Overall Performance 2D vs. 3D Pain Drawing.

This is generally in line with our expectations. In fact, although opinions about the importance of recording pain information and the usefulness of doing so across time were roughly the same when using both the 2D and 3D drawings, surprisingly, despite the age group of some of the participants, it was found that using the $3 \mathrm{D}$ drawing on the laptop was easier than using the paper-based, 2D version (Fig. 4a). Nevertheless, we assume that younger patients will not have these same concerns, a condition that is significantly encouraging with respect to the results.

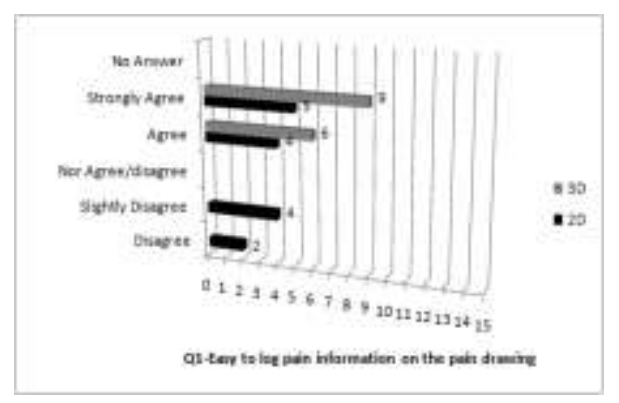

(a)

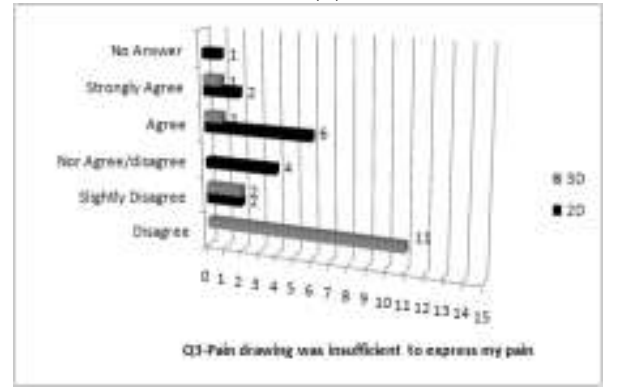

(c)

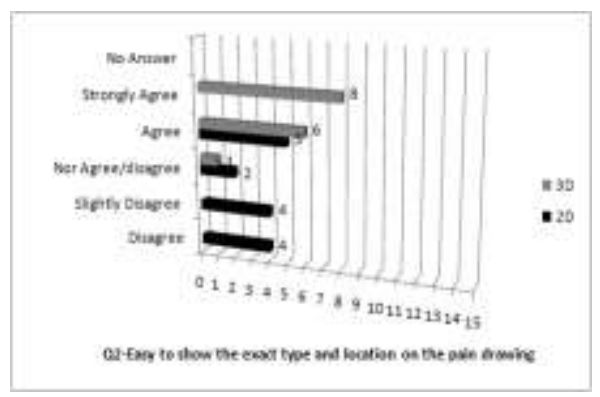

(b)



(d)

Fig. 4. Bar Graphs of Responses to Evaluation Questions. 
Specifically, considering that some participants suffered from mobility issues due to the nature of their SCI, it was rational to assume that they would encounter difficulties in using the 3D user interface on the laptop; however, in overall they agreed that the process was a relatively easy one and that the 3D pain drawing was indeed beneficial.

The results with respect to the developed 3D interface's feasibility to visualize pain are also particularly encouraging (Fig. 4b). Considering the limited abilities that the 2D drawing was offering, it comes as no surprise that patients found that showing the exact type and location of their pain on the 3D pain drawing was significantly easier than when using the $2 \mathrm{D}$ one.

Lastly, our research study has revealed that the general trend was that patients were enthusiastic about the 3D pain drawing, highlighting the wide acceptability and approval of the 3D model's ability to more sufficiently visualize their pain experience (Fig. 4c). In specific, the majority of the SCI patients that participated in our study appreciated the advantages of the enhanced visualization ability that our 3D model provides by indicating very positive views towards its overall interface layout (Fig. $4 d)$.

\section{Conclusions}

This study has presented a novel solution for better describing and communicating SCI pain characteristics, which was developed by exploiting the possibilities offered by the advancements in 3D visualization technology. The motivation behind our work lays in the fact that the current pain assessment methods are considered inadequate in their attempt to provide the necessary assistance to the individual with SCI in more accurately describing the pain experience.

As a result, our cohort evaluation with 15 participants, recruited from an Orthopedic Hospital in London, UK, has demonstrated that it is feasible to apply 3D visualization technology in order to more sufficiently assess pain resulting from SCI. In specific, the results of our work have revealed that SCI patients valued the ability of the 3D pain drawing to describe their pain to the medical staff involved in their rehabilitation, by making positive comments, such as "the $2 D$ drawing was not adequate..", highlighting in this way the insufficiency of its $2 \mathrm{D}$ predecessor.

Moreover, it is anticipated that the use of such technology creates the possibility for patients to become stakeholders in the management of their pain, by allowing first to communicate their pain experience to clinicians in a more perceivable way to the natural environment, and second, to use this visualization ability in order to further understand it. Finally, this study has demonstrated that with the continuous advancements in 3D technology, clinical applications that would utilize such 3D functionality could become an integral part in the rehabilitation of people with some form of disabling pain. 


\section{References}

1. Broeren, J, Sunnerhagen, K.S., Rydmark, M.: Haptic virtual rehabilitation in stroke: transferring research into clinical practice. Physical Therapy Reviews, 14 (5), 322-335 (2009).

2. Ciocca, L., Fantini, M., Marchetti, C., Scotti, R., Monaco, C.: Immediate facial rehabilitation in cancer patients using CAD-CAM and rapid prototyping technology: a pilot study. Support Care Cancer, 18, 723-728 (2010).

3. Coll, A.M., Ameen, J.R.M., Mead, D.: Postoperative pain assessment tools in day surgery: literature review. Journal of Advanced Nursing, 46 (2), 124-133 (2004).

4. Felix, E.R., Cruz-Almeida, Y., Widerstrom-Noga, E.G.: Chronic pain after spinal cord injury: What characteristics make some pains more disturbing than others? Journal of Rehabilitation Research and Development, 44 (5), 703-716 (2007).

5. Felix, E.R., Galoian, K.A., Aarons, C., Brown, M.D., Kearing, S.A., Heiss, U.: Utility of quantitative computerized pain drawings in a sample of spinal stenosis patients. Pain Medicine, 11(3), 382-389 (2010)

6. Ghinea, G., Spyridonis, F., Serif, T., Frank, A.O.: 3-D Pain Drawings-Mobile Data Collection Using a PDA. IEEE Transactions on Information Technology in Biomedicine, 12(1), 27-33 (2008).

7. Haefeli, M., Elfering, A.: Pain assessment. European Spine Journal, 15, 17-24 (2006).

8. Jamison, R.N., Fanciullo, G.J., Baird, J.C.: Usefulness of Pain Drawings in Identifying Real or Imagined Pain: Accuracy of Pain Professionals, Non professionals, and a Decision Model. The Journal of Pain, 5 (9), 476-482 (2004).

9. Lee, S.J.: Pain measurement: Understanding existing tools and their application in the emergency department. Emergency Medicine, 13, 279-287 (2001).

10.Mannion, A.F., Balague, F., Pellise, F., Cedraschi, C.: Pain measurement in patients with low back pain. Nature Clinical Practice Rheumatology, 3 (11), 610-618 (2007).

11.Mooney, V., Cairns, D., Robertson, J.: A system for evaluating and treating chronic back disability. West J. Med. 124, 370-376 (1976).

12.Ohnmeiss, D.D.: Repeatability of Pain Drawings in a Low Back Pain Population. Spine, 25 (8), 980-988 (2000).

13.Provenzano, D.A., Fanciullo, G.J., Jamison, R.N., McHugo, G.J., Baird, J.C.: Computer Assessment and Diagnostic Classification of Chronic Pain Patients. Pain Medicine, 8 (S3), 167-175 (2007).

14. Ransford, A. O, Cairns, D., Mooney, V.: The Pain Drawing as an Aid to Psychologic Evaluation of Patients with Low-Back pain. Spine, 1 (2), 127-134 (1976).

15. Samuelsson, K., Larsson, H., Thyberg, M., Tropp, H.: Back Pain and Spinal DeformityCommon Among Wheelchair Users with Spinal Cord Injuries. Scandinavian Journal of Occupational Therapy, 3(1), 28-32 (1996).

16. Siddall, P.J., Yezierski, R.P., Loeser, J.D.: Pain Following Spinal Cord Injury: Clinical Features, Prevalence, and Taxonomy. IASP Press, IASP Newsletter 3, Seattle (2000).

17.Spinal Injuries Association (SIA): Impact Reports and Financial Statements, http://www.spinal.co.uk/page/Reports-and-Accounts (2009)

18. Takata, K., Hirotani, H.: Pain drawing in the evaluation of low back pain. International Orthopaedics, 361-366 (1995).

19.Wang, M.Y., O’Shaugnessy, B., Haq, I., Green, B.A.: Pain Following Spinal Cord Injury. Seminars in Neurosurgery, 15 (1), 99-105 (2004). 